\title{
A system for remote recognition of emotions from a facial expression
}

\author{
G.R. Khazankin ${ }^{1,2 *}$, A. Malinin², I. Shmakov ${ }^{2}$ \\ ${ }^{1}$ Scientific-Research Institute of Physiology and Basic Medicine, Novosibirsk, Russia \\ ${ }^{2}$ Novosibirsk State University, Novosibirsk, Russia \\ *e-mail:khazankin@nsuacad.net
}

Key words: facial expression, arousal, valence, psychophysiology, machine learning, data streams

\begin{abstract}
Motivation and Aim: Nowadays social networks are a commonplace platform for communication of many people. Users create web content, check out the content of others and send private messages. Network communication enables people to control their actions carefully, while anonymity allows them to act without reservations about getting unclosed. Therefore, it is interesting to find the relation between the real psychophysiological state of users and their various actions. This paper describes a method for collecting information of user's emotional state based on its facial expressions and linking the data acquired to the user's actions in a time.
\end{abstract}

Methods and Algorithms: At client side, we use reactive programming model implemented by RxJS library to manage data streams. Our client library includes logic to combining events from different sources such as MediaStream APIs, classic DOM event API. We have developed our own neural network architecture that allows us to map the facial expressions of people into a continuous space of arousal/valence [1]. The same architecture for classification of emotions was used. We used inception and residual blocks and batch normalization layers to achieve higher accuracy. In addition, we augment train data by adding pose variations using 3D face reconstruction [2].

Results: We have developed a system that allows us to collect various data such as video stream and events of user interaction with the webpage. We also trained a neural network that shows high accuracy. One part of the system is a client library that does not require the user to install any special software and can be easily integrated into the website. The other part is a server application that enables the site owner to analyze the data collected. Conclusion: The developed system allows us to collect the necessary data about users remotely and in settings familiar for people. The next steps in our study are to collect a large amount of data and compare the actions of users with their psychophysiological state. Besides, we aim to try to extract other user characteristics such as heart rate and sentiment from deleted text messages.

\section{References}

1. Schroff F., Kalenichenko D., Philbin J. (2015) Facenet: A unified embedding for face recognition and clustering. Proceedings IEEE Conference Computer Vision Pattern Recognition:815-823.

2. Jackson A.S., Bulat A., Argyriou V., Tzimiropoulos G. (2017) Large pose 3D face reconstruction from a single image via direct volumetric CNN regression. Computer Vision (ICCV), 2017 IEEE International Conference on:1031-1039. 